\title{
A Compact Threshold-Voltage Model of MOSFETs with Stack High- $k$ Gate Dielectric
}

\author{
F. Ji, J. P. Xu*, J. J. Chen, H. X. Xu, C. X. Li, P. T. Lai*
}

\begin{abstract}
In this paper, a compact threshold-voltage model is developed for stack high- $k$ gate-dielectric MOSFET with a thin interlayer. The simulated results are in good agreement with 2-D simulations. The influences of $k$ value of the interlayer on threshold behaviors are investigated in detail. A low- $k$ interlayer can effectively improve the threshold-voltage behaviors. Furthermore, the ratio of low- $k$ interlayer EOT (equivalent oxide thickness) to high- $k$ layer EOT is optimized by considering both threshold-voltage roll-off and gate leakage current.
\end{abstract}

Keywords: MOSFET, Threshold voltage, stack gate dielectric

\section{INTRODUCTION}

High- $k$ gate dielectric has become a promising candidate for future nano-scaled MOSFETs. However, both high- $k / \mathrm{Si}[1,2]$ and high $-k / \mathrm{Ge}[3,4]$ interfaces suffer from unacceptably high interface-state density, thus resulting in low carrier mobility. So, a thin interlayer, e.g. SiON for Si MOS [5] and $\mathrm{TaO}_{\mathrm{x}} \mathrm{N}_{\mathrm{y}}$ for Ge MOS [6], is needed to improve the interface properties, and thus stack gate-dielectric MOS devices with thin interlayer become attractive.

The threshold voltage of MOSFET with a single-layer high- $k$ gate dielectric has been studied widely $[7,8]$, and however, threshold behaviors of the stack high- $k$ gate-dielectric MOSFET are less addressed. In this work, for the first time, a threshold-voltage model of the stack gate-dielectric MOSFET is developed, and the influences of $k$ value of the thin interlayer on surface potential and threshold voltage roll-off are discussed in detail. It is found that threshold behaviors of the stack-gate dielectric MOSFET can be improved by using a low- $k$ interlayer, and a suitable ratio of equivalent oxide thickness (EOT) of the interlayer to EOT of the

F. Ji is with Department of Electronic Science and Technology, Huazhong University of Science and Technology, and also with Wuhan Institute of Technology, Wuhan 430074, People's Republic of China

J. P. Xu, J J Chen, and H X Xu are with Department of Electronic Science and Technology, Huazhong University of Science and Technology, Wuhan 430074, People's Republic of China

*E-mail: ipxu@mail.hust.edu.cn

C. X. Li, and P. T. Lai are with Department of Electrical \& Electronic Engineering, the University of Hong Kong, Pokfulam Road, Hong Kong

*E-mail: laip@eee.hku.hk high- $k$ dielectric layer is required to reduce both threshold-voltage roll-off and gate leakage current.

\section{MODEL DERIVATION}

A schematic diagram of the stack high- $k$ gate-dielectric MOSFET with a thin interlayer is shown in Fig.1, where $T_{o x 1}$ and $T_{o x 2}$ are high- $k$ dielectric thickness and interlayer thickness respectively (corresponding EOT is denoted as EOT1 and EOT2); $T_{o x}$ $=T_{o x l}+T_{o x 2}$ is total gate-dielectric thickness, and $x_{d}$ is the depletion-region width in the substrate.

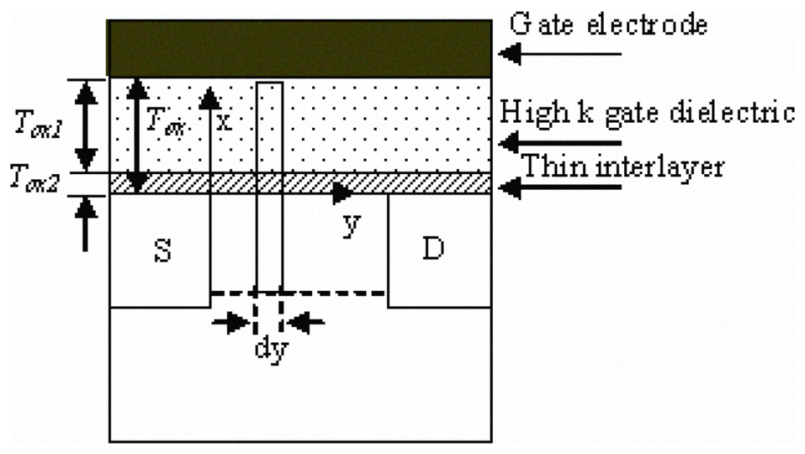

Fig. 1 Schematic diagram of MOSFET with stack high- $k$ gate dielectric.

For simplicity, the polysilicon depletion effect and quantum effects are neglected. So, the 2-D Poisson's equation in the depletion region and stack gate-dielectric region can be written as.

$$
\frac{\partial^{2} \varphi(x, y)}{\partial x^{2}}+\frac{\partial^{2} \varphi(x, y)}{\partial y^{2}}= \begin{cases}0, & T_{o x 2} \leq x \leq T_{o x}, \\ 0, & 0 \leq x \leq T_{o x 2}, \\ q N_{A} / k_{s u b}, & -x_{d} \leq x \leq 0,\end{cases}
$$

where $N_{A}$ is the doping concentration of p-type substrate; $k_{\text {sub }}$ is the permittivity of the substrate. The solution of Eq. (1) can be found to be $[7,8]$ :

$$
\begin{aligned}
\varphi(x, y)= & \varphi_{0}(x)+\left[\varphi(x, 0)-\varphi_{0}(x)\right] \frac{\sinh [(L-y) / l]}{\sinh (L / l)} \\
& +\left[\varphi(x, L)-\varphi_{0}(x)\right] \frac{\sinh (y)}{\sinh (L / l)}
\end{aligned}
$$

in which $L$ is the channel length; $l$ is defined as characteristic length; $\varphi_{0}(x)$ is the solution of the 1-D 
Poisson's equation for a long-channel MOSFET.

By applying the Gauss's law to a rectangular box [Gaussian box: dy $\times\left(x_{d}+T_{o x}\right)$ ] from the bottom of the depletion region to the top of the high $k$ dielectric layer via the interlayer, as shown in Fig.1, the characteristic length can be derived as:

$$
l^{2}=\frac{k_{s u b} x_{d}}{C_{o x}}+\frac{\int_{0}^{T_{o x}} k(x)\left[\varphi(x, 0)-\varphi_{0}(0)\right] d x}{C_{o x}\left[\varphi(0,0)-\varphi_{0}(0)\right]}
$$

where $k(\mathrm{x})$ is the relative permittivity somewhere between $\mathrm{x}=0$ and $x=T_{o x}, C_{o x}$ is total gate capacitance. Using boundary conditions: $\varphi(x, 0)=V_{b i}$ and $\varphi(x, L)=V_{b i}+V_{d s}\left(\mathrm{~V}_{\mathrm{bi}}\right.$ and $\mathrm{V}_{\mathrm{ds}}$ are the built-in potential of drain-substrate junction and the drain-source voltage, respectively), $l$ can be written as:

$$
l^{2}=\frac{k_{s u b} x_{d}}{C_{o x}}+\frac{C_{o x}\left(\frac{k_{o x 2}}{k_{o x 1}} T_{o x 1} T_{o x 2}+\frac{T_{o x 1}^{2}+T_{o x 2}^{2}}{2}\right)}{C_{o x}}
$$

where $k_{o x l}$ and $k_{o x z}$ are the relative permittivity of the high- $k$ dielectric and interlayer respectively. Based on Liu's [7] and our previous work [8], the threshold voltage can be expressed as:

$$
V_{t h}=V_{t h 0}-\frac{\left\lfloor 2\left(V_{b i}-2 \phi_{f}\right)+V_{d s}\right\rfloor}{2 \cosh (L / 2 l)-2}
$$

in which $V_{t h 0}$ is threshold voltage of 1-D long-channel MOSFET. Threshold-voltage roll-off resulted from the short-channel effect (SCE) and fringing-field effect (FFE) is defined as:

$$
V_{t h}-V_{t h 0}=-\frac{\left[2\left(V_{b i}-2 \phi_{f}\right)+V_{d s}\right]}{2 \cosh (L / 2 l)-2}
$$

\section{RESULTS AND DISCUSSION}

Eq. (5) shows that the threshold voltage is related to the characteristic length, and the larger the characteristic length, the stronger is the FFE. Change of the characteristic length of Si MOSFET with the

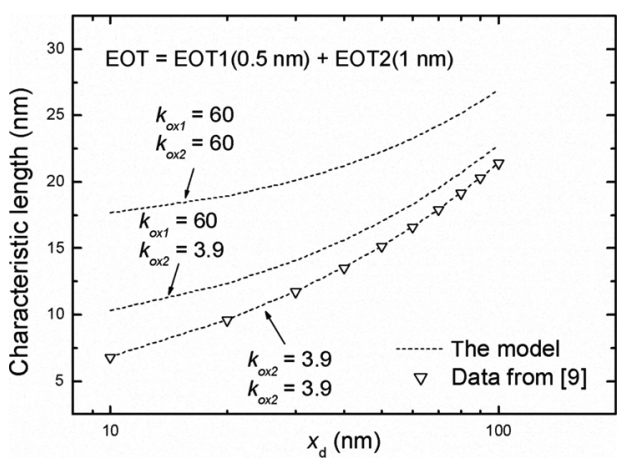

Fig. 2 Characteristic length versus $x_{d}$ for different $k_{o x l}$ and $k_{o x 2}$ depletion-region width is depicted in Fig.2 for different gate-stack structures. For Si MOSFET with $\mathrm{SiO}_{2}$ as gate dielectric, the simulated results exhibit good agreement with that in [9], thus confirming the validity of the model. At a given $x_{d}$, the characteristic length for $k_{o x l}=k_{o x 2}=60$ (i.e. single-layer high-k gate dielectric) is larger than that for $k_{o x l}=k_{o x 2}=3.9$ (i.e. single-layer $\mathrm{SiO}_{2}$ gate oxide), implying a strong FFE in high- $k$ MOSFET. Also, it can be seen that the $l$ of MOSFET with stack gate dielectric of $\mathrm{SiO}_{2}$ as interlayer is close to that of MOSFET with single $\mathrm{SiO}_{2}$ as gate dielectric, meaning that the $\mathrm{SiO}_{2}$ interlayer plays an important role in alleviating the FFE of the stack high- $k$ gate-dielectric MOSFET.

Surface potential of both $\mathrm{Si}$ and Ge MOSFETs with single and stack gate dielectric is plotted in Fig.3 (a) and (b) respectively. For the MOSFET with single gate dielectric, it can be seen that the surface potential of the MOSFET is higher for $k_{o x l}=k_{o x 2}=60$ than $k_{o x l}=k_{o x 2}=$ 3.9. However, the surface potential of the stack-gate dielectric MOSFET with $k_{o x l}=60$ and $k_{o x 2}=3.9$ is slightly higher than that of MOSFET with $k_{o x l}=k_{o x 2}=$ 3.9 , and much lower than that of MOSFET with $k_{o x l}=$ $k_{o x 2}=60$. Compared with single high- $k$ gate-dielectric MOSFET, the stack gate-dielectric MOSFET with low- $k$ interlayer can alleviate the influence of the fringing-field effect on channel surface potential, thus giving better threshold voltage behaviors.

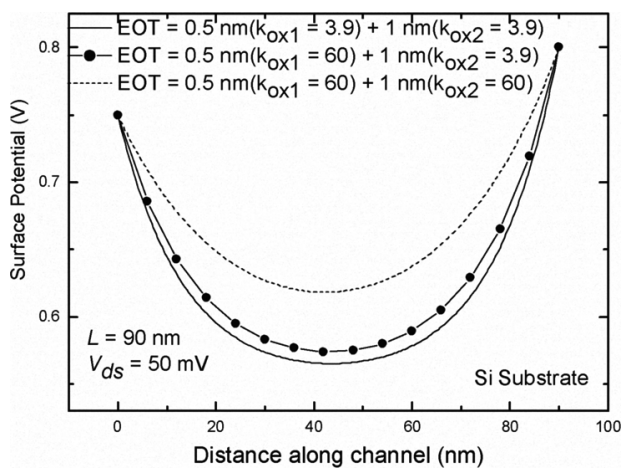

(a) Si substrate

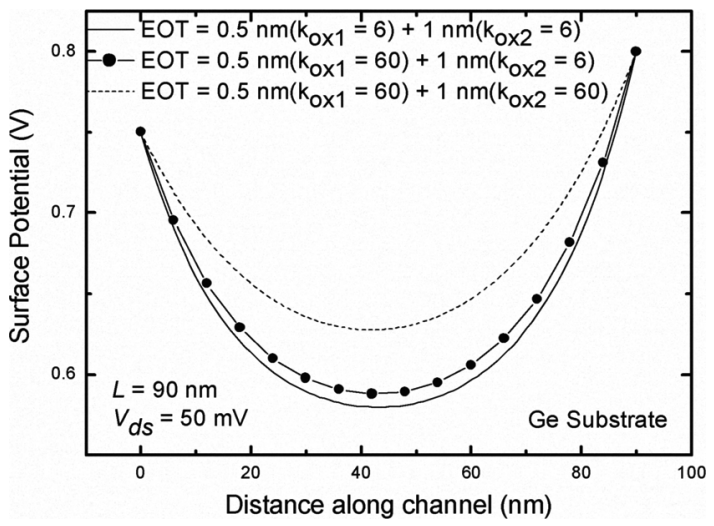

(b) Ge substrate

Fig. 3 Surface potential of (a) Si and (b) Ge MOSFETs with single and stack gate dielectric.

Fig.4 shows the dependence of threshold-voltage roll-off on channel length for different gate-dielectric 
structures. As $L$ decreases, the threshold voltages of $\mathrm{Si}$ and Ge MOSFETs are decreased due to the enhanced fringing-field effect and short-channel effect. For MOSFET with single gate dielectric, good agreement of this model with 2-D simulation [7] verifies the validity of this model. Compared to single high- $k$ gate-dielectric MOSFET with $k_{o x 1}=k_{o x 2}=60$, threshold-voltage roll-off of the stack gate-dielectric MOSFET with $k_{o x l}=60$ and $k_{o x 2}=3.9$ is closer to that of MOSFET with single $\mathrm{SiO}_{2}$ as gate dielectric, indicating the fringing-field effect of small-scaled MOSFET can be effectively suppressed by inserting a low- $k$ interlayer between the high- $k$ gate dielectric and substrate. The above results show this analytical model can describe the short-channel effect and fringing-field effect of MOSFET with stack-gate dielectric.

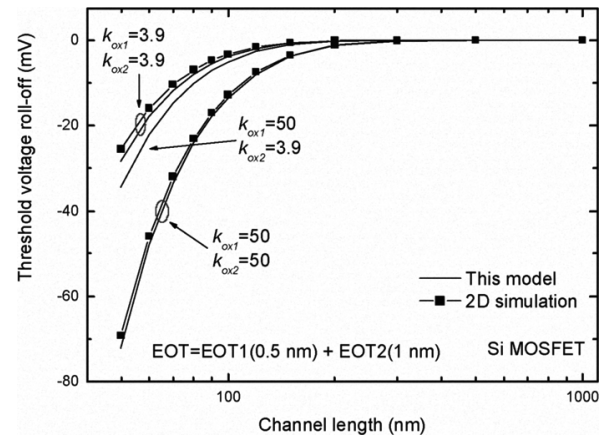

(a) Si MOSFET

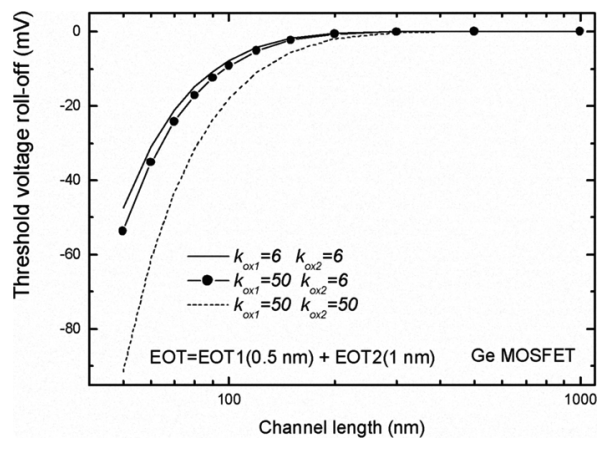

(b) Ge MOSFET

Fig. 4 Threshold-voltage roll-off of (a) $\mathrm{Si}$ and (b) $\mathrm{Ge}$ MOSFET with single and stack gate dielectric versus channel length.

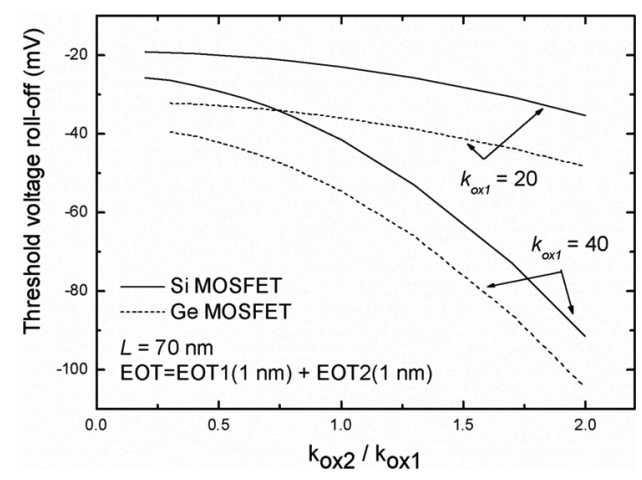

Fig.5 Dependence of threshold-voltage roll-off on $k_{o x 2} /$ $k_{\text {oxl }}$
For a given $k_{o x l}$, as the ratio of $k_{o x 2} / k_{o x 1}$ increases, the threshold-voltage roll-off is increased (as seen in Fig. 5 ), which shows a low- $k$ interlayer with good interface quality with $\mathrm{Si}\left(\right.$ e.g. $\mathrm{SiO}_{2}$ ) or $\mathrm{Ge}$ (e.g. $\left.\mathrm{GeON}\right)$ should be used to improve the threshold-voltage properties.

For two different double-layer gate dielectric stacks, as shown in Fig. 6(a), setting EOT1 $=1 \mathrm{~nm}$, EOT2 $=1$ $\mathrm{nm}$ and total EOT $=2 \mathrm{~nm}$, the simulated results show that much better threshold-voltage behavior is obtained for the case 1 than the case 2, due to electric field divergence on the top low- $k$ dielectric layer for case 2 [9], as can be seen in Fig. 6(b). This implies that a stable metal gate electrode, which would not react with the underlying high- $k$ dielectric layer to produce low- $k$ interlayer at metal/high-k interface, should be used to improve the threshold-voltage behaviors of MOSFETs.

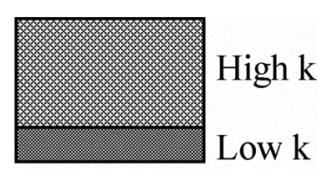

Case 1

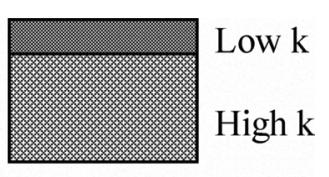

Case 2 (a)

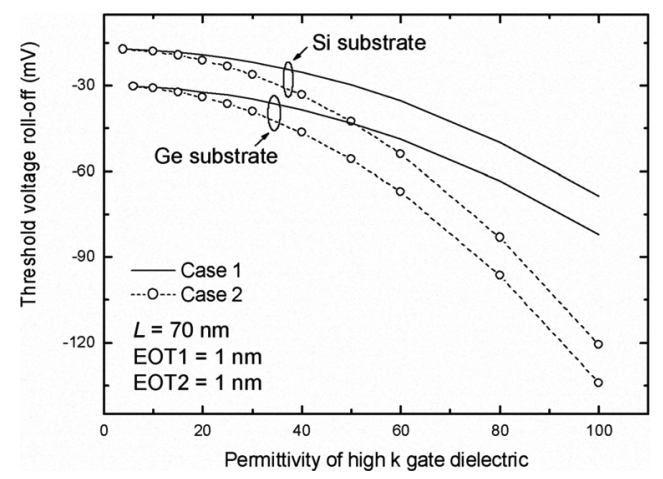

(b)

Fig. 6 (a) Two schematic stack gate dielectrics, and (b) threshold-voltage roll-off of corresponding MOSFETs.

Influence of low- $k$ interlayer thickness on threshold-voltage roll-off of MOSFET with stack-gate dielectric EOT of $2 \mathrm{~nm}$ is shown in Fig.7. The threshold-voltage roll-off at EOT1 $=0 \mathrm{~nm}$ and EOT2 $=2$ $\mathrm{nm}$ (i.e. single-layer low- $k$ gate dielectric) is mainly resulted from the short-channel effects. For both $\mathrm{Si}$ and Ge MOSFETs, as EOT2 decreases (EOT1 increases), threshold-voltage roll-off is increased due to enhancement of fringing-field effect induced by the high-k gate dielectric. So, the thicker the EOT2, the smaller is the threshold-voltage roll-off. On the other hand, larger EOT2 results in smaller total physical thickness of gate dielectric for a given EOT, which would give rise to a larger gate leakage current. Gate leakage current of MOS device with $\mathrm{SiO}_{2}$ interlayer and $\mathrm{HfO}_{2}(k \approx 25)$ high- $k$ layer was calculated for different EOT1/EOT2 and fixed total EOT of $2 \mathrm{~nm}$ [10]. Although gate leakage current is below $10^{-9} \mathrm{~A} / \mathrm{cm}^{2}$ at $V_{g}=-1 \mathrm{~V}$ for EOT1/EOT2 $=1.75 \mathrm{~nm} / 0.25 \mathrm{~nm}$ [10], threshold-voltage 
roll-off is large, as calculated by the above model (solid square symbol in Fig.7). Contrarily, for EOT1/EOT2 = $0.25 \mathrm{~nm} / 1.75 \mathrm{~nm}$, a good threshold behavior is achieved $\left(\Delta \mathrm{V}_{\text {th }} \approx 17 \mathrm{mV}\right.$, solid circle symbol in Fig.7), but the gate leakage current density $\left(J_{g}\right)$ is larger than $10^{-3} \mathrm{~A} / \mathrm{cm}^{2}$ at $V_{g}=-1 \mathrm{~V}$, which cannot meet the requirement of $J_{g}<10^{-3}$ $\mathrm{A} / \mathrm{cm}^{2}$ [11]. Therefore, the ratio of EOT1/EOT2 needs to be optimized for stack gate dielectric. This can be realized by considering a trade-off between gate leakage current and threshold-voltage behaviors. For example, $J_{g}$ is calculated to be $<10^{-4} \mathrm{~A} / \mathrm{cm}^{2}$ [10] for EOT1/EOT2 of $0.5 \mathrm{~nm} / 1.5 \mathrm{~nm}$ to $1.25 \mathrm{~nm} / 0.75 \mathrm{~nm}$, while threshold-voltage roll-off is $18 \sim 22 \mathrm{mV}$, closed to the value for EOT1/EOT2 $=0.25 \mathrm{~nm} / 1.75 \mathrm{~nm}(\sim 17 \mathrm{mV})$, as shown by the open up-triangle and open down-triangle symbols in Fig. 7. Thus, the EOT1/EOT2 $=0.5 \mathrm{~nm} / 1.5$ $\mathrm{nm}$ to $1.25 \mathrm{~nm} / 0.75 \mathrm{~nm}$ would be a reasonable choice for gate stack structure.

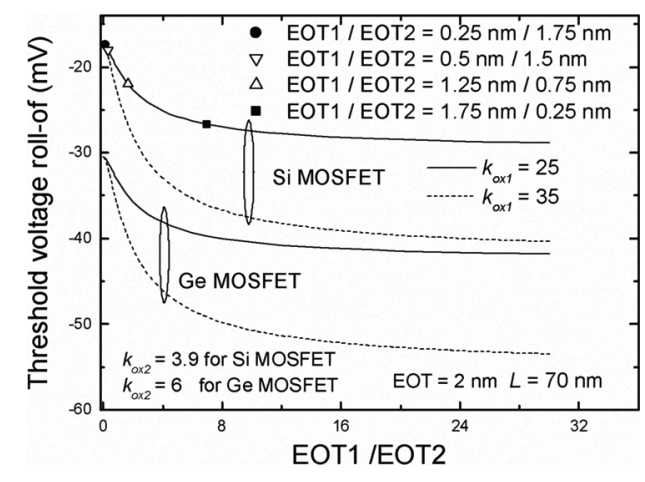

Fig.7 Threshold-voltage roll-off as a function of EOT1/EOT2

\section{CONCLUSION}

An analytical threshold-voltage model suitable for both $\mathrm{Si}$ and Ge MOSFETs with stack gate dielectric is derived. Influences of the $k$ value of interlayer on threshold properties are discussed in detail. It is found that when the interlayer is a low- $k$ dielectric, e.g. $\mathrm{SiO}_{2}$, or $\mathrm{GeON}$, the threshold behaviors of the stack high- $k$ gate-dielectric MOSFET could be improved, but on the contrary, the threshold-voltage roll-off of the MOSFET is increased. Therefore, optimization of the EOT1/EOT2 ratio is required to obtain both small threshold-voltage roll-off and low gate leakage current.

\section{ACKNOWLEDGEMENTS}

This work is financially supported by the National Natural Science Foundation of China under Grant 60776016, and the RGC of HKSAR, China (Project No. HKU 713308E) and Foundation of Hubei Provincial Department of Education (Grant no. Q20095001).

\section{REFERENCES}

[1] S.A. Campbell, D.C. Gilmer, X.C. Wang, M.T. Hsieh H.S. Kim, W. L. Gladfelter, and j. Yan, "MOSFET transistors fabricated with high permittivity $\mathrm{TiO}_{2}$ dielectrics," IEEE Trans. Electron Devices, vol.44, p.104, Jan 1997.

[2] I.C. Kizilyalli, P.K. Roy, F.Baumann, R.Y.Huang, D.Hwang, C. Chacon, R. Irwin, Y. Ma, and G. Alers, "Stacked gate dielectrics with $\mathrm{TaO}$ for future CMOS technologies," in Proc. VLSI Symp. Technology, p.216, 1998.

[3] Xiao Zou, and Jing-Ping $\mathrm{Xu}$, "Study on electrical properties of HfTiON and HfTiO gate dielectric Ge MOS capacitors with wet-NO surface pretreatment," IEEE international conference on, Solid-state and integrated-circuit technology, p. 1280 , Oct. 2008.

[4] X. F. Zhang, J. P. Xu, C. X. Li, P. T. Lai, C. L. Chan, and J. G. Guan, "Improved electrical properties of $\mathrm{Ge}$ metal-oxide-semiconductor capacitor with HfTa-based gate dielectric by using $\mathrm{TaO}_{\mathrm{x}} \mathrm{N}_{\mathrm{y}}$ interlayer," Applied Physics Letters, vol. 92 p.262902, Jun 2008.

[5] F. Ji, J. P. Xu, P. T. Lai, C. X. Li, and J. G. Guan, "Electrical properties of HfTiON gate-dielectric metal-oxide-semiconductor capacitors with different Si-surface nitridations," Applied physics letters, vol.91 p.052902, 2007.

[6] J. P. Xu, X. F. Zhang, C. X. Li, P. T. Lai and C. L. Chan, "Improved Electrical Properties of $\mathrm{Ge}$ p-MOSFET with $\mathrm{HfO}_{2}$ Gate Dielectric by Using $\mathrm{TaO}_{\mathrm{x}} \mathrm{N}_{\mathrm{y}}$ Interlayer," IEEE Electron Device Letters, vol.29(10), p.1155, Oct, 2008.

[7] Xiaoyan Liu, Jifeng Kang, Ruqi Han, and Yangyuan Wang, "threshold voltage model for MOSFETs with high-k gate dielectrics," IEEE Electron Device Letters, vol.23 (5), p.270, May 2002.

[8] J. P Xu, F. Ji, P. T. Lai and J. G. Guan, "Influence of sidewall spacer on threshold voltage of MOSFET with high-k gate dielectric," Microelectronics Reliability, vol.48, p.181, 2008.

[9] Zhi-Hong Liu, Chenming Hu, Jian-Hui Huang, Tung-Yi Chan, Min-Chie Jeng, Ping K. Ko, and Y. C. Cheng, "Threshold voltage model for deep-submicrometer MOSFET's," IEEE Transactions on electron devices, vol.40 (1), p.86, 1993.

[10] W. B. Chen, J. P. Xu, P. T. Lai, Y. P. Li, and S. G. $\mathrm{Xu}$, "Gate leakage properties of MOS devices with tri-layer high-k gate dielectric," Microelectronic reliability, vol. 47, p.937, 2007

[11] H. R. Huff, A. Hou, C. Lim, Y. Kim, J. Barnett, G. Bersuker, G. A. Brown, C. D. Young, P. M. Zeitzoff, J. Gutt, P. Lysaght, M. I. Gardner, R. W. Murto, "High-k gate stacks for planar, scaled CMOS integrated circuits," vol. 69, p.152, 2003. 